\title{
Tracheostomy in the COVID-19 pandemic
}

\author{
Francesco Mattioli ${ }^{1}$ (1) $\cdot$ Matteo Fermi ${ }^{1} \cdot$ Michael Ghirelli $^{1} \cdot$ Gabriele Molteni $^{3} \cdot$ Nicola Sgarbi $^{2} \cdot$ Elisabetta Bertellini $^{2}$. \\ Massimo Girardis ${ }^{2} \cdot$ Livio Presutti $^{1} \cdot$ Andrea Marudi $^{2}$
}

Received: 7 April 2020 / Accepted: 11 April 2020 / Published online: 22 April 2020

(c) Springer-Verlag GmbH Germany, part of Springer Nature 2020

\begin{abstract}
Purpose The role of tracheostomy in COVID-19-related ARDS is unknown. Nowadays, there is no clear indication regarding the timing of tracheostomy in these patients.

Methods We describe our synergic experience between ENT and ICU Departments at University Hospital of Modena underlining some controversial aspects that would be worth discussing tracheostomies in these patients. During the last 2 weeks, we performed 28 tracheostomies on patients with ARDS due to COVID-19 infection who were treated with IMV. Results No differences between percutaneous and surgical tracheostomy in terms of timing and no case of team virus infection.

Conclusion In our experience, tracheostomy should be performed only in selected patients within 7- and 14-day orotracheal intubation.
\end{abstract}

Keywords COVID-19 · Tracheostomy $\cdot$ Invasive mechanic ventilation

To date, over 940,000 “Coronavirus Disease 2019” (COVID19) cases have been confirmed, with over 39,000 deaths. At the writing time, the COVID-19 epidemic is striking hard in Italy, being the second country in terms of infected and dead people. The mortality appears to be around $2 \%$; early published data indicate $25.9 \%$ with COVID-19 pneumonia required intensive care unit (ICU) admission and 20.1\% developed acute respiratory distress syndrome (ARDS) [1, 2]. Non-invasive support methods (i.e., CPAP, BiPAP, NIV, HFNO) might correct hypoxemia and avoid endotracheal intubation but may either delay intubation with potential complications. Invasive mechanical ventilation (IMV) via an endotracheal tube (ETT) is being commonly employed during this outbreak.

Francesco Mattioli

franz318@hotmail.com

1 Otolaryngology-Head and Neck Surgery Department, University Hospital of Modena, Via del Pozzo 71, 41100 Modena, Italy

2 Department of Anesthesiology, University Hospital of Modena, Via del Pozzo 71, 41100 Modena, Italy

3 Head and Neck Department, University of Verona, Verona, Italy
Tracheostomy is a widely used intervention in patients with acute respiratory failure, especially when clinicians predict a patient's need for prolonged IMV, but at the moment, no recommendation on COVID-19-affected patients exists [3].

Herein, we describe our synergic experience between ENT and ICU Departments at University Hospital of Modena underlining some controversial aspects that would worth discussing tracheostomies in these patients.

During the last 2 weeks, we performed 28 tracheostomies on patients with ARDS due to COVID-19 infection who were treated with IMV.

There are no studies addressing mechanical ventilation strategies in COVID-19 outbreaks. COVID-19-related ARDS often requires prolonged intubation as observed in our experience. In addition, early extubating attempts frequently require a re-intubation procedure. According to Meng et al. these should be considered difficult and complicated intubations for different reasons (i.e., no respiratory reserve, strict infection control and urgency, personal protective equipment, psychological pressure) [4].

Tracheostomy offers several advantages in terms of improved comfort management, reduced sedative, and paralytic medical support, reducing death space. Moreover, 
tracheostomy reduces airways resistance, lessening the work of breathing optimizing the tracheal secretion control [5].

This procedure results in better patient comfort and facilitates restoring patient autonomy, even if it carries its own risks. Aerosol-generating procedures, such as endotracheal intubation, extubation, and tracheostomy, have been implicated with the transmission of infectious agents to healthcare personnel in previous papers regarding the SARS outbreak.

An increasing need for ICU admission due to COVID19-related ARDS can be expected within the next months. The magnitude of this demand may cause an imbalance between the real clinical needs of the population and the effective availability of intensive resources. This scenario can be substantially assimilated to the field of "Disaster medicine" where tracheostomy is a tool for early ICU discharge [3]. Indeed, tracheostomy could allow discharge from ICU to intermediate care ward in patients with ongoing IMV.

In the LUNG SAFE study, involving ARDS patients from 459 ICUs across 50 countries, tracheostomy was performed in $13 \%$ of patients, whose $75 \%$ after the first week of ICU stay [6]. The median timing of tracheostomy was 14 days after the onset of ARDS. The difficulty for clinicians in predicting which patients will require prolonged ventilation support in the early phases of critical illness is a caveat. There is no identified time point when afflicted patients either improve, remain stable, or progress toward death due to pulmonary complications.

No recommendation can be given about tracheostomy within 7 days in COVID-19 patients. In this early phase, aggressive treatments and intensive care are needed for critically ill patients and tracheostomy could not result in improvement in hypoxia, multiple organ dysfunction, virus clearance, and in shorter duration of IMV.

If the patient meets weaning targets like $\mathrm{FiO} 2$ less than $40 \%, \mathrm{PEEP}<8, \mathrm{PaO} 2 / \mathrm{FiO} 2>200$, pressure support $<8$ $\mathrm{cmH}_{2} \mathrm{O}$, extubation could be reached between 7 and 14 days, tracheostomy should be postponed.

After 7 days in patients far from reaching weaning targets, tracheostomy could be performed, but there is a lack of data regarding this topic.

Additional critical outcome scores, such as high respiratory SOFA score, might be beneficial to identify those COVID-19 patients who are too sick to benefit from further interventional procedure [7].

Moreover, performing a tracheostomy appears to be influenced by other factors as local medical practices and expertise and costs relating to the procedure and equipment.

In our experience, several delayed ( $>14$ OTI days) tracheostomies were performed especially in the first-affected intubated cases who required re-intubation without clinical improvement, and in case of lack of ICU places. We report a case where important tracheomalacia and posterior tracheal wall damage were observed after the opening of the trachea, probably caused by prolonged over-cuffed intubation and/or prone position ventilation or previously repeated extubation and re-intubation procedures. We did not delay tracheostomy timing until the COVID-19 deactivation time was reached and no one has been infected. However, unless emergent, surgical procedures should only be undertaken after ascertaining the COVID-19 status to decrease potential virus exposure to the team.

It is recommended during aerosol-generating procedures on patients with COVID-19 to wear a fit-tested N95 mask in addition to gloves, gown, face/eye protection and to perform these procedures in an airborne isolation room. Both percutaneous and surgical tracheostomies can be performed. Surgical tracheostomy should be indicated for BMI $>30$ patients with short neck and documented thyroid gland hypertrophy. We have been always performing trans-isthmus tracheostomy with tracheal-toskin stitches on inferior margin without ETT removal to avoid accidental displacement of tracheal cannula. Cuffed non-fenestrated cannula should be used to limit diffusion of the virus. Every effort should be made not to pierce the cuff of the ETT when performing tracheostomy. Initial advancement of the ETT should be performed prior to tracheostomy window being made. Ventilation has to cease prior to tracheostomy tube insertion, which has to be swift and accurate with prompt inflation of the cuff. Most skilled ENT surgeon should perform the procedure due to the uncomfortable setting of the ICU COVID-19 room and personal protective equipment.

The role of tracheostomy in COVID-19-related ARDS is unknown. Nowadays, there is no clear indication regarding the timing of tracheostomy in these patients. There is no evidence that tracheostomy improves patient's clinical course. It is established that tracheostomy reduces the ICU stay. In the context of prolonged IMV required in COVID19 experience, tracheostomy should be suggested to avoid potential tracheal damages within 7 and 14 days. Surgical tracheostomy should be proposed only in selected patients.

The ENT and ICU specialists should pay meticulous attention to the details of infection control, to minimize cross-contamination and their own risk of contracting the illness. We suggest other colleagues to share their experience with this topic.

Funding None.

\section{Compliance with ethical standards}

Conflict of interest The authors declare that they have no conflict of interest.

Ethical approval Not applicable. 
Informed consent Not applicable.

\section{References}

1. Lai C-C, Shih T-P, Ko W-C, Tang H-J, Hsueh P-R (2020) Severe acute respiratory syndrome coronavirus 2 (SARS-CoV-2) and coronavirus disease-2019 (COVID-19): the epidemic and the challenges. Int J Antimicrob Agents 55(3):105924. https://doi. org/10.1016/j.ijantimicag.2020.105924

2. Coronavirus. https://www.who.int/emergencies/diseases/novel -coronavirus-2019. Accessed 25 Mar 2020.

3. Alhazzani W, Møller MH, Arabi YM et al (2019) Surviving sepsis campaign: guidelines on the management of critically ill adults with coronavirus disease 2019 (COVID-19). Intensive Care Med 2020:104

4. Meng L, Qiu H, Wan L et al (2020) Intubation and ventilation amid the COVID-19 outbreak: Wuhan's experience. Anesthesiology. https://doi.org/10.1097/ALN.0000000000003296

5. Vergano M, Bertolini G, Giannini A, et al. (2020) Clinical ethics recommendations for the allocation of intensive care treatments, in exceptional, resource-limited circumstances. Italian Society of Anesthesia, Analgesia, Resuscitation, and Intensive Care (SIAARTI). https://www.siaarti.it/SiteAssets/News/COVID 19\%20-\%20documenti\%20SIAARTI/SIAARTI\%20-\%20Cov id-19\%20-\%20Clinical\%20Ethics\%20Reccomendations.pdf. Accessed 16 Mar 2020

6. Bellani G, Laffey JG, Pham T et al (2016) Epidemiology, patterns of care, and mortality for patients with acute respiratory distress syndrome in intensive care units in 50 countries. JAMA 315(8):788-800. https://doi.org/10.1001/jama.2016.0291

7. Vincent JL, Moreno R, Takala J et al (1996) The SOFA (Sepsis-related Organ Failure Assessment) score to describe organ dysfunction/failure. On behalf of the Working Group on SepsisRelated Problems of the European Society of Intensive Care Medicine. Intensive Care Med 22(7):707-710. https://doi.org/10.1007/ bf01709751

Publisher's Note Springer Nature remains neutral with regard to jurisdictional claims in published maps and institutional affiliations. 